\title{
TEAPOT-SPINK simulation of orbits and spin in COSY with an RF-solenoid (January 2011 runs)
}

\author{
A.U. Luccio, N. Malitsky, Y. Semertzidis, J.D.Talman \\ Brookhaven National Laboratory
}

\author{
E. Stephenson \\ Indiana University
}

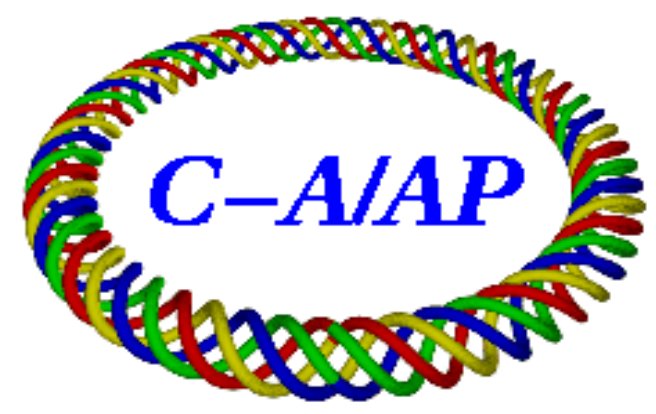

\section{Collider-Accelerator Department Brookhaven National Laboratory Upton, NY 11973}

Notice: This document has been authorized by employees of Brookhaven Science Associates, LLC under Contract No. DE-AC02-98CH10886 with the U.S. Department of Energy. The United States Government retains a nonexclusive, paid-up, irrevocable, world-wide license to publish or reproduce the published form of this document, or allow others to do so, for United States Government purposes. 


\title{
TEAPOT-SPINK simulation of orbits and spin in COSY with an RF-Solenoid (January 2011 runs)
}

\author{
A.U.Luccio ${ }^{1 *}$, N.Malitski ${ }^{1}$, Y.Semertzidis ${ }^{1}$, E.Stephenson ${ }^{2}$, J.D.Talman ${ }^{1}$ \\ ${ }^{1}$ Brookhaven National Laboratory, Upton, NY 11973, USA, \\ ${ }^{2}$ Indiana Univ., Bloomington, IN, USA \\ *E-mail: luccio@bnl.gov
}

July 31, 2013

Presented in "slide" format to the German Physical Society (DPG) "Meeting on Search for Electric Dipole Moments (eDMs) at Storage Rings"

Bad Honnef, Germany July 4-6, 2011

\begin{abstract}
After outlining the main strategy for orbit and spin simulation for the proposed pEDM storage ring project[1] at BNL, we present a first set of results of the simulation with the library UAL(TEAPOTSPINK)[3] of the experiments performed at COSY, to study in detail methods of spin polarimetry for the pEDM.
\end{abstract}




\section{Tracking and experiments for the pEDM}

Orbit and spin tracking for the pEDM proton storage ring experiment is important to:

- Track particle with longitudinal spin polarization for a large number of orbits, to detect the appearance of a vertical spin component that will indicate the presence of an electric dipole moment.

- Estimate spin coherence time of a polarized beam and study correction of the same by using non linear optics elements, like sextupoles.

The strategy we are pursuing at BNL is to use at least two competitive computer approaches, based on different algorithms, namely

1. Numerical integration of the equations of orbital motion and spin precession: RK4 [?, ?] and integration by series: Zgoubi [2].

2. Thin element kick propagation, via the Unified Accelerator Library, UAL[?]ontaining TEAPOT [6], for orbits, and SPINK [4] for spin.

The first set of algorithms is generally slower and then less apt for long term tracking, but may produce a more detailed representation of the behavior of the protons in all lattice elements of the ring, in particular through numerical field maps. The second method is faster and apt to represent beam and spin behavior in a very large number of turns.

It is important to mention that for spin simulation at BNL on the storage ring RHIC[5] a similar approach is being pursued using TEAPOT-SPINK (through UAL) and Zgoubi as complementary methods, with results from the two codes continuously cross-checked in weekly meetings.

Experiments to test and validate equipment and procedures for the proposed storage ring are also essential.

On this line, experiments in January 2011 at the storage ring COSY at the German Research center of Jlich have been performed to address issues of spin polarimetry on a working machine in condition similar to what it will be at the pEDM

We are simulating with TEAPOT-SPINK the results of the experiments with the dual purpose of tracking on a well established lattice and benchmark the codes, that will be then used for the pEDM experiment. 
This contribution is a status report of the presently ongoing simulation of COSY by TEAPOT-SPINK, as a continuation, with improved algorithms, of the preliminary study of spin coherence in COSY with the classical SPINK, that was published earlier [7].

\section{The RF solenoid}

The Thomas-BMT equation for relativistic spin motion in a magnetic field can be written as

$$
\frac{d \mathbf{s}}{d t}=-\frac{q}{m \gamma} \mathbf{a} \times \mathbf{s}
$$

with $\mathbf{s}$ the spin vector and

$$
\mathbf{a}=(1+G) \mathbf{B}_{\|}-(1+G \gamma) \mathbf{B}_{\perp}
$$

where $\mathbf{B}_{\|}$and $\mathbf{B}_{\perp}$ are the magnetic field components parallel and perpendicular to the particle orbit, respectively.

In an RF Solenoid the field is parallel: $\mathbf{B} \equiv \mathbf{B}_{\|}$, then Eq.(1) become simply

$$
\frac{d \mathbf{s}}{d t}=-\frac{q}{m \gamma}(1+G) \mathbf{B} \times \mathbf{s} .
$$

Write explicitly for the components of s: x(radial), $\mathrm{y}$ (vertical), $\mathrm{z}$ (longitudinal)

$$
\frac{d \mathbf{s}}{d t}=-\frac{q}{m \gamma}(1+G)\left(\begin{array}{ccc}
\hat{i} & \hat{j} & \hat{k} \\
S_{x} & S_{y} & S_{z} \\
0 & 0 & B
\end{array}\right)=-\frac{q}{m \gamma}(1+G) B\left[\hat{i} S_{y}-\hat{j} S_{x}\right]
$$

or

$$
\left\{\begin{array}{l}
\frac{d s_{x}}{d t}=-\frac{q}{m \gamma}(1+G) B s_{y} \\
\frac{d s_{y}}{d t}=\frac{q}{m \gamma}(1+G) B s_{x}
\end{array}\right.
$$

after another differentiation and back substitution

$$
\left\{\begin{array}{l}
\frac{d^{2} s_{x}}{d t^{2}}+\lambda^{2} s_{x}=0 \\
\frac{d^{2} s_{y}}{d t^{2}}+\lambda^{2} s_{y}=0
\end{array}, \quad \text { with } \quad \lambda=\frac{q}{m \gamma}(1+G) B\right.
$$


The integral is

$$
\left\{\begin{array}{l}
s_{x}=s_{x}^{(0)} \cos (\lambda t)+s_{y}^{(0)} \sin (\lambda t) \\
s_{y}=-s_{x}^{(0)} \sin (\lambda t)+s_{y}^{(0)} \cos (\lambda t)
\end{array} .\right.
$$

From either Eq.(5), in a thin solenoid of thickness $d \ell=\beta c d t$ the spin angular kick is

$$
d \mathbf{s}=-\frac{q}{m c \beta \gamma}(1+G) \mathbf{B} d \ell \times \mathbf{s} .
$$

It is also

$$
B \rho=10^{9} \frac{p c}{c}, \quad p c=\beta \gamma m c^{2}, \quad \text { then }: \quad \frac{q}{m c \beta \gamma}=\frac{1}{B \rho}
$$

with the rigidity of the beam $B \rho$ in [T.m], and $p c$ and the rest energy $m c^{2}$ in $[\mathrm{GeV}]$. It is also in the RF Solenoid

$$
B=B_{0} \sin \left(\int \omega_{R F} d t\right)
$$

From Eqs.(8),(9),(10) we can write

$$
\lambda=\lambda_{0} \sin \left(\int \omega_{R F} d t\right), \quad \text { with } \quad \lambda_{0}=\frac{B_{0} d \ell}{B \rho}(1+G)
$$

A search for Froissart-Stora transition frequency, where the RF Solenoid induces a spin flip, yields the experimental result, that theoretically is at

$$
\omega_{R F}=(1 \pm G \gamma) \omega_{c y}, \quad \text { with the cyclotron frequency } \omega_{c y}=\frac{2 \pi}{T_{0}}=\frac{2 \pi \beta c}{L}
$$

where $T_{0}$ is the period of revolution and $L$ the lenght of the accelerator ring. 
Use:

$$
\beta \gamma=\frac{p c}{m c^{2}}, \quad \gamma=\sqrt{1+(\beta \gamma)^{2}}, \quad \beta=\sqrt{1-\frac{1}{\gamma^{2}}},
$$

Numbers for COSY and the COSY RF solenoid, runs of January 2011, are (courtesy of Bernd Lorentz and Ed Stephenson):

$\begin{array}{lll}\mathrm{c} & =2.997923210^{8}[\mathrm{~m} / \mathrm{s}] \\ \text { particle } & =\text { deuteron } \\ m c^{2} & =1.87562799[\mathrm{GeV}] \\ G & =-0.14298727 \\ \rho & =7[\mathrm{~m}] \\ p c & =0.97061778[\mathrm{GeV}] \\ \gamma, \beta & =1.12596420,0.45959675 \\ B \rho & =3.2356166[\mathrm{~T} \cdot \mathrm{m}] \\ Q_{x}, Q_{y} & =3.61,3.619 \\ L, T_{0} & =183.473[\mathrm{~m}], 1.33077318[\mu \mathrm{s}] \\ \omega_{c y} & =4.72145473[\mathrm{MHz}] \\ B d \ell & =0.0005 \mathrm{~T} \cdot \mathrm{m} \\ \lambda_{0} & =1.296509510^{-4}\left[\mathrm{~s}^{-1}\right] \\ \omega_{R F} / \omega_{c},(\mp) & =1.16099855,0.83900145[\mathrm{MHz}] \\ 1[\mathrm{~s}] \text { of run } & =751,443 \text { turns }\end{array}$

\section{Orbit}

In the simulation the orbit of COSY is calculated by UAL-TEAPOT, by a sequence of symplectic kicks. The elements of the ring, read from an sxf file created by MADX, are subdivided into slices to insure a required accuracy. The code well compares with other tracking codes based on the integration of differential equations, but is comparatively faster.

In the present exercise we are using the COSY lattice with parameters used in the experimental runs we want to simulate,containing multi function bending magnets and their fringing fields, quadrupoles, sextupoles, a RF cavity and the RF Solenoid (assumed for this case to be of zero length). A typical transverse orbit, turn by turn obtained by tracking is shown in Fig.1

The longitudinal motion bucket with an $R F$ cavity set at $\mathrm{V}=400 \mathrm{~V}$, harmonic $\mathrm{h}=1$, is shown in Fig.2. 


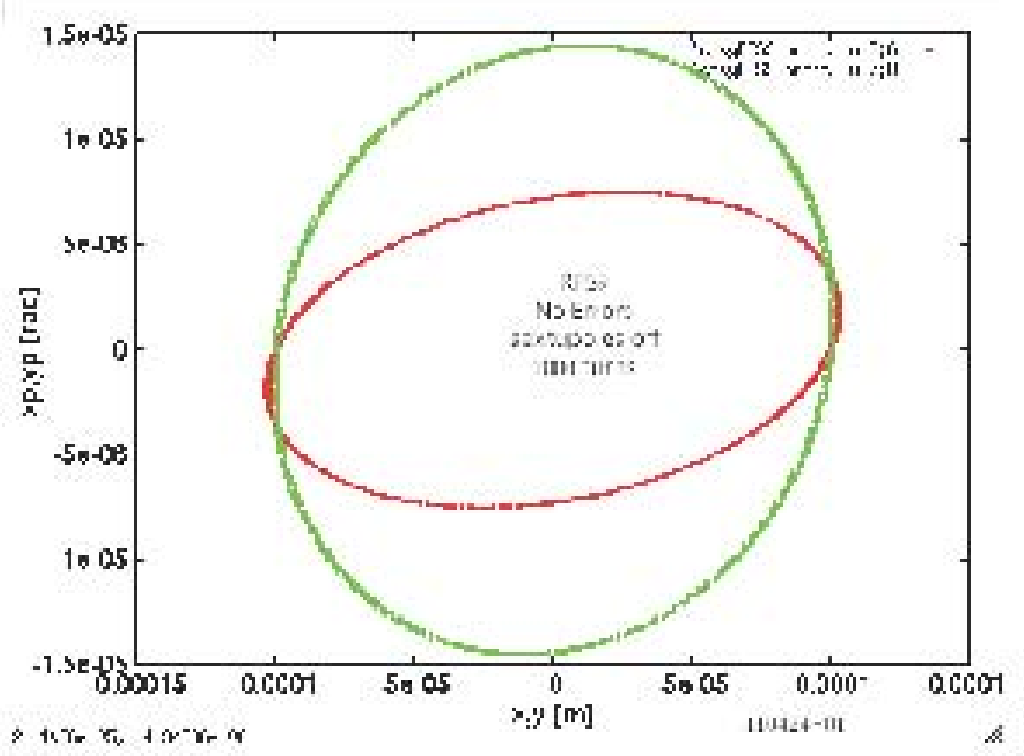

Figure 1: Transverse orbit, turn-by-turn. Initial $\mathrm{x}$ and $\mathrm{y}=0.1 \mathrm{~mm}$

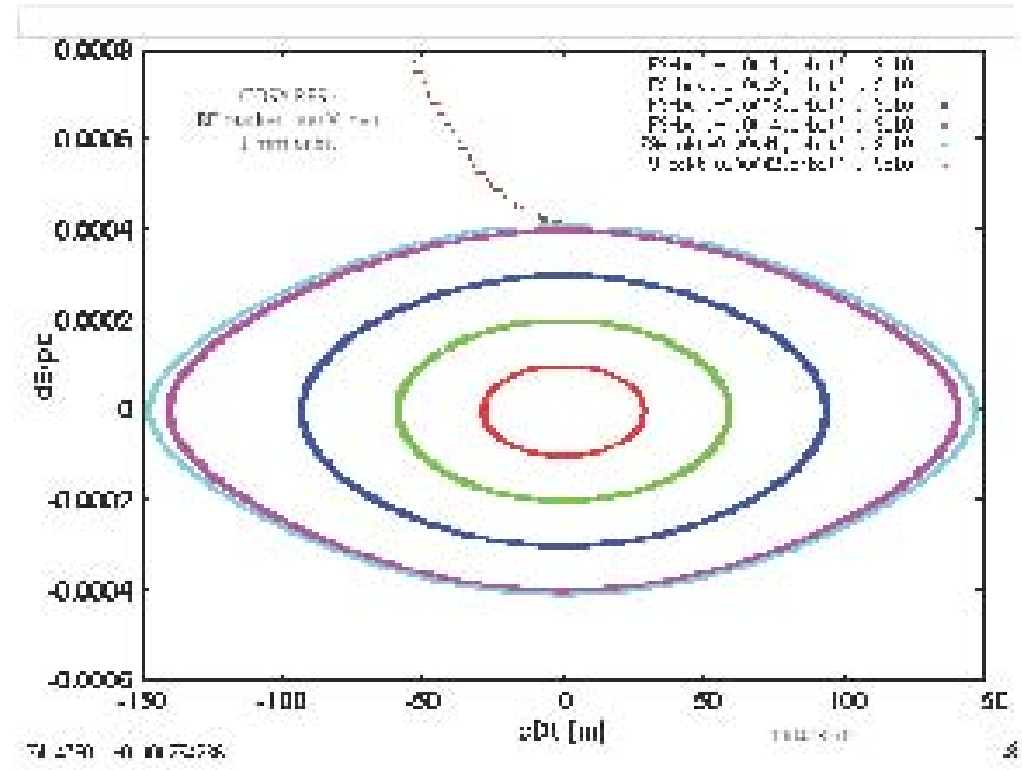

Figure 2: Transverse RF bucket, to the limits of its stability 


\section{Spin}

In the simulation the orbit of COSY is calculated by UAL-SPINK, by a sequence of angle kicks according to the Thomas-BMT Equation, with an algorithm that preserves the integrity of the spin vector and represents a precession of the spin around a local axis. The code uses the same partitions of the machine elements as TEAPOT does for the orbit. The spin is rotated in the bends, in the bend fringes, and in all multipoles present in the machine lattice, Receiving periodic kicks by the RF solenoid.

From the eigenvalues of the one-turn spin matrix, the spin tune is calculated, resulting close to the reference value $G \gamma$. For this, see the discussion on spin tune line and spin tune coherence in a later section of this paper

With the RF Solenoid freely running we simulate a polarization curve measured in the experiment, shown in Fig.3

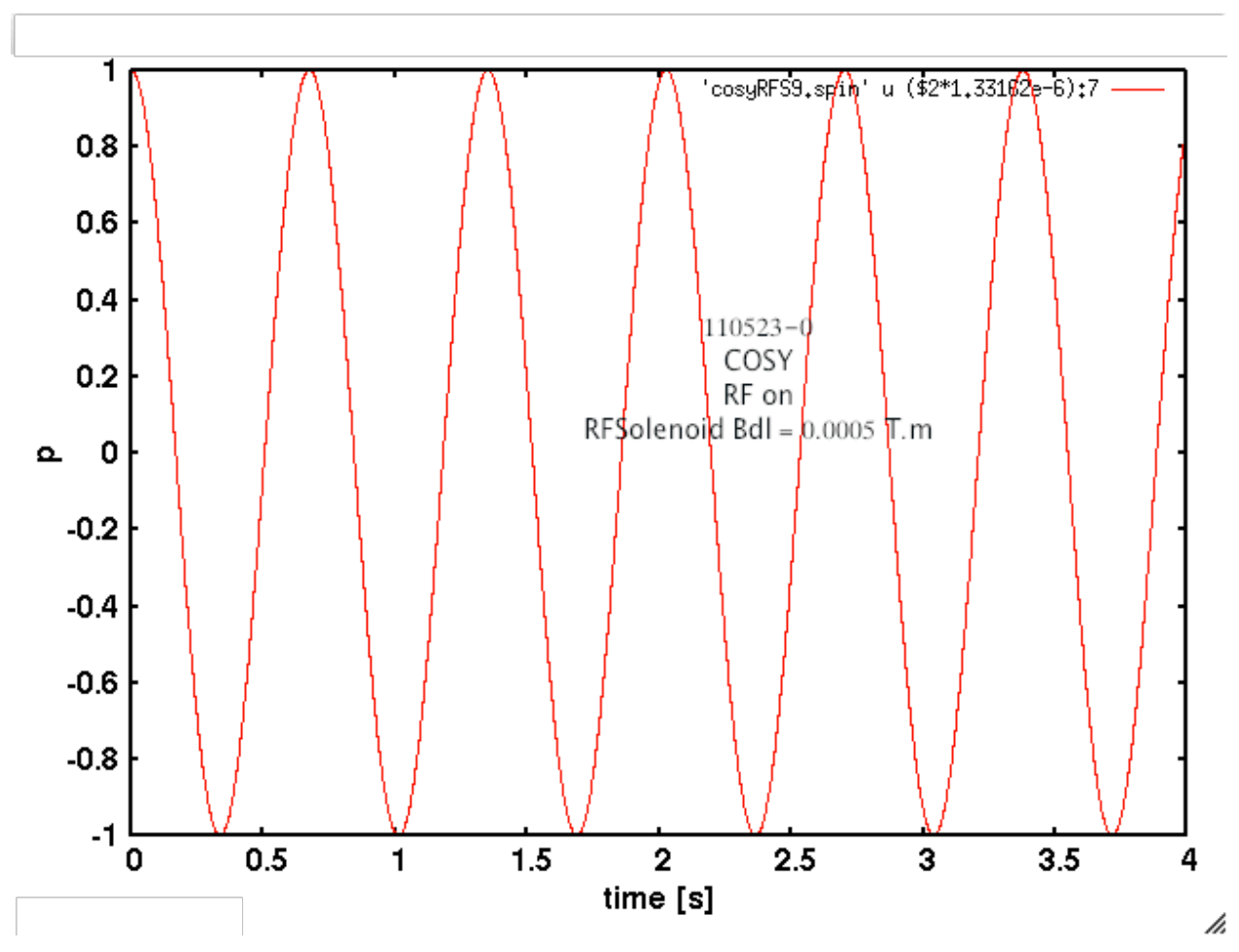

Figure 3: example caption 


\section{Froissart-Stora}

We tried to simulate the spin flip at the resonance induced by the RF solenoid by ramping linearly its frequency at different speed. The results are shown in Fig.4. The spin flip should have occurred at the frequency $(1-G \gamma) f_{c y}=$ $1.160996 f_{c y}$ according to Eq.(12), instead it appears at $1.160986 f_{c y}$, lower by a factor 1.e-6. We have not yet discovered the source of this small discrepancy.

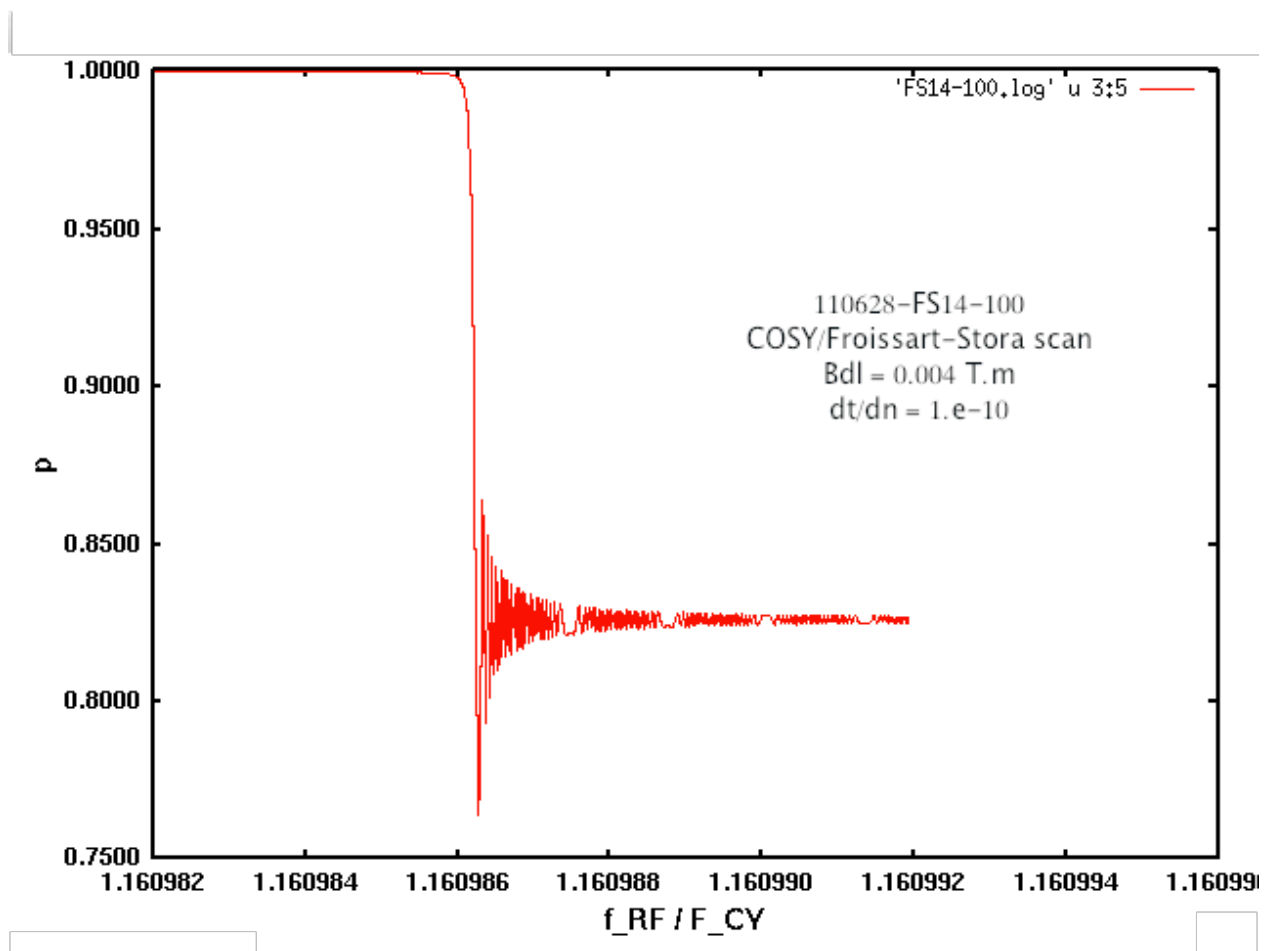

Figure 4: Froissart-Stora partial spin flip due a linear ramp of the frequency of the RF Solenoid.Ramp speed in this example was $\delta f /$ turn $=1.10^{-10}$ and solenoid strength 0.0004 T.m. Vertical polarization is shown vs. RF solenoid frequency. 


\section{Spin Tune}

Spin tune, or number of spin precession per machine turn, is calculated as an eigenvalue of the one-turn spin $3 \times 3$ matrix OT. Since the spin oscillations from one orbit turn to the next can slightly vary because the orbital phase space vector takes many turns to regain its starting value, the spin tune is equivalently calculated by running average over many turns.

Calling $T=O T_{11}+O T_{22}+O T_{33}$ the trace of the spin matrix, the fractional part of spin tune $Q_{\text {spin }}$ is

$$
\tilde{Q}_{\text {spin }}=\frac{\mu}{2 \pi}, \quad \mu=\arccos \left(\frac{1-T}{2}\right) .
$$

The above is only able to calculate the fractional part or its complement to one, depending on the sign of $\mu$, that can be determined from the two Euler angles of the orientation of the spin precession axis, $\theta$, latitude, and $\phi$, longitude. These angles are in turn calculated from other elements of the one turn spin matrix, as follows

$$
\left\{\begin{array}{c}
\phi=\arctan (2)\left(\left(O T_{12}-O T_{21}\right),\left(O T_{23}-O T_{32}\right)\right) \\
\theta=\arctan (2)\left(\left(O T_{23}+O T_{32}\right) \sin \phi,\left(O T_{13}+O T_{31}\right)\right) \\
\sin \mu=\frac{O T_{12}-O T_{21}}{2 \cos \mu} \\
\operatorname{sign}(\mu)=-\arctan (2)(\sin \mu, \cos \mu)
\end{array}\right.
$$

and

$$
\operatorname{if}(\operatorname{sign}(\mu)<=1)\left\{\tilde{Q}_{\text {spin }}=1-\tilde{Q}_{\text {spin }} \cdot\right\}
$$

Examples of spin tune for the present COSY lattice are shown in the following figures. Fig. 5 shows the spin tune running average over 15,000 turns for a single particle injected on axis, at 1, 2, 5,10, $20 \mathrm{~mm}$ from the ring axis, both on the horizontal and vertical direction. For this example the RF cavity was not activated and the momentum of the particle was on the reference value.

The spin tune of the particle at $x=y=0$ is exactly

$$
Q_{\text {spin }}=G \gamma=0.16097792
$$

as it should (horizontal line on the plot). Values for the other particles are 


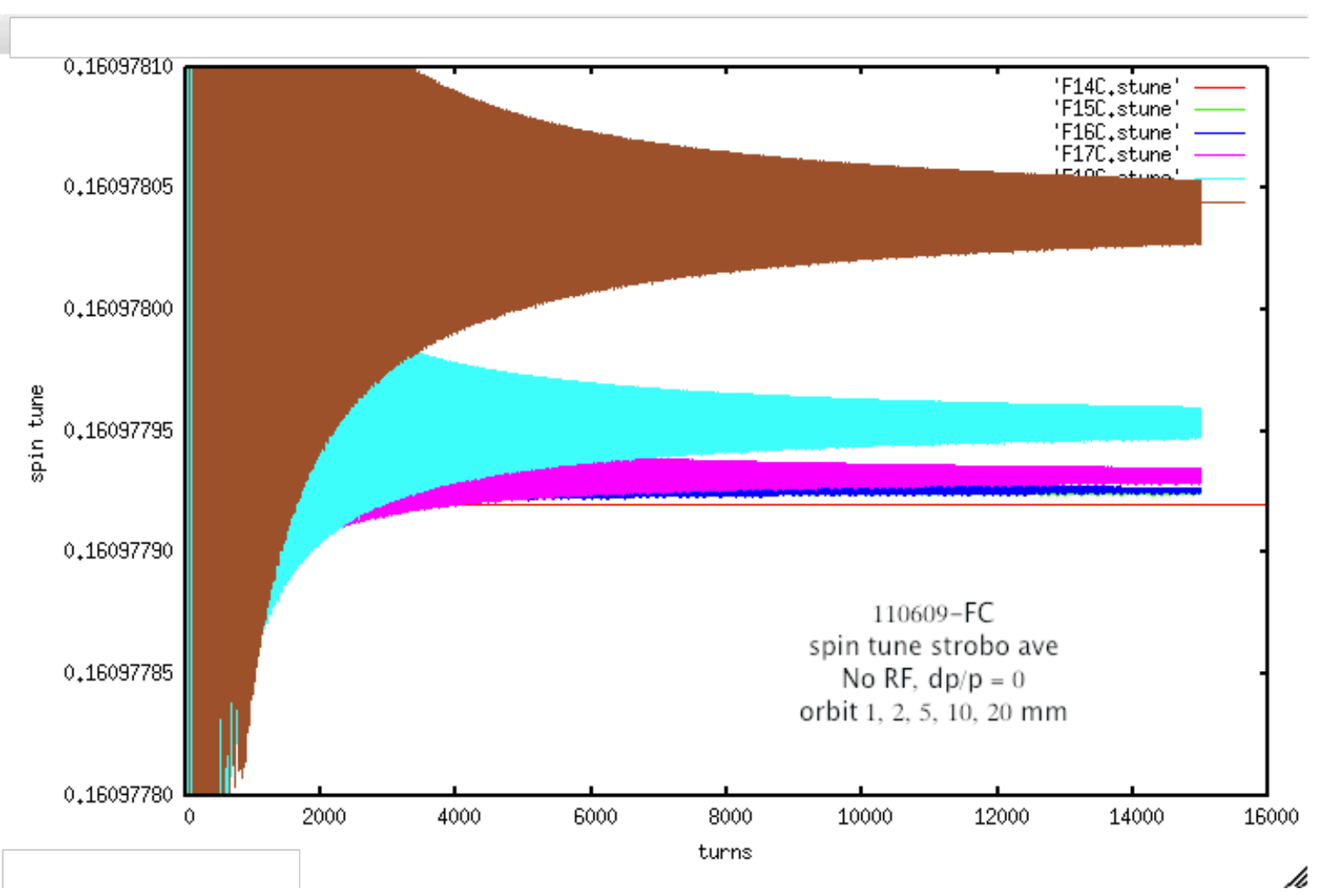

Figure 5: No RF, $d p / p=0$, orbit at $0,1,2,5,10,20 \mathrm{~mm}$ 


\begin{tabular}{lll} 
to Fig.5 & $Q_{\text {spin }}$ \\
\hline $0 \mathrm{~mm}$ & $:$ & 0.16097792338328884 \\
$1 \mathrm{~mm}$ & $:$ & 0.16097792470682865 \\
$2 \mathrm{~mm}$ & $:$ & 0.16097792589360971 \\
$5 \mathrm{~mm}$ & $:$ & 0.16097793267073540 \\
$1 \mathrm{~cm}$ & $:$ & 0.16097795551645017 \\
$2 \mathrm{~cm}$ & $:$ & 0.16097804475330965 \\
$5 \mathrm{~cm}$ & $:$ & 0.16097866586279944 \\
\hline
\end{tabular}

The largest difference in spin tune in this table is $7.510^{-7}$, with an average spin tune line width of the order of $10^{-7}$, corresponding to a spin coherence of the order of $10^{7}$ turns, only due to transverse orbital motion.

We repeated the same exercise for a particle with no orbital motion, still no RF, but with increasing momentum deviation from the reference particle. Results are:

\begin{tabular}{lll}
$\begin{array}{l}\text { No orbital motion } \\
\mathrm{dp} / \mathrm{p}\end{array}$ & \\
\hline $1.10^{-4}$ & $:$ & 0.16097792338328884 \\
$2.10^{-4}$ & $:$ & 0.16097792425088700 \\
$5.10^{-4}$ & $:$ & 0.16097792634534910 \\
$1.10^{-3}$ & $:$ & 0.16097793393329327 \\
$2.10^{-3}$ & $:$ & 0.16097796567275011 \\
$5.10^{-3}$ & $:$ & 0.16097818589420571 \\
$1.10^{-2}$ & $:$ & 0.16097558395455833 \\
\hline
\end{tabular}

\section{$7 \quad$ Spin coherence}

Spin coherence is a property of the entire particle bunch in the machine. Polarization of the bunch is proportional to its spin coherence. A bunch is spin coherent if all particle spin precession remain in phase for a certain time.

The largest difference in spin tune in the tables is $7.610^{-6}$, with an average spin tune line width of the order of $10^{-6}$, corresponding to a spin coherence of the order of $10^{6}$ turns, only due to energy spread in the rather large interval here considered.

We turned on the RF cavity in COSY, to allow oscillations in the longitudinal phase space shown in Fig.2 of energy and phase. Figs.6 and 7 show the effect of synchrotron oscillations on the spin tune stroboscopic averaging pattern. 


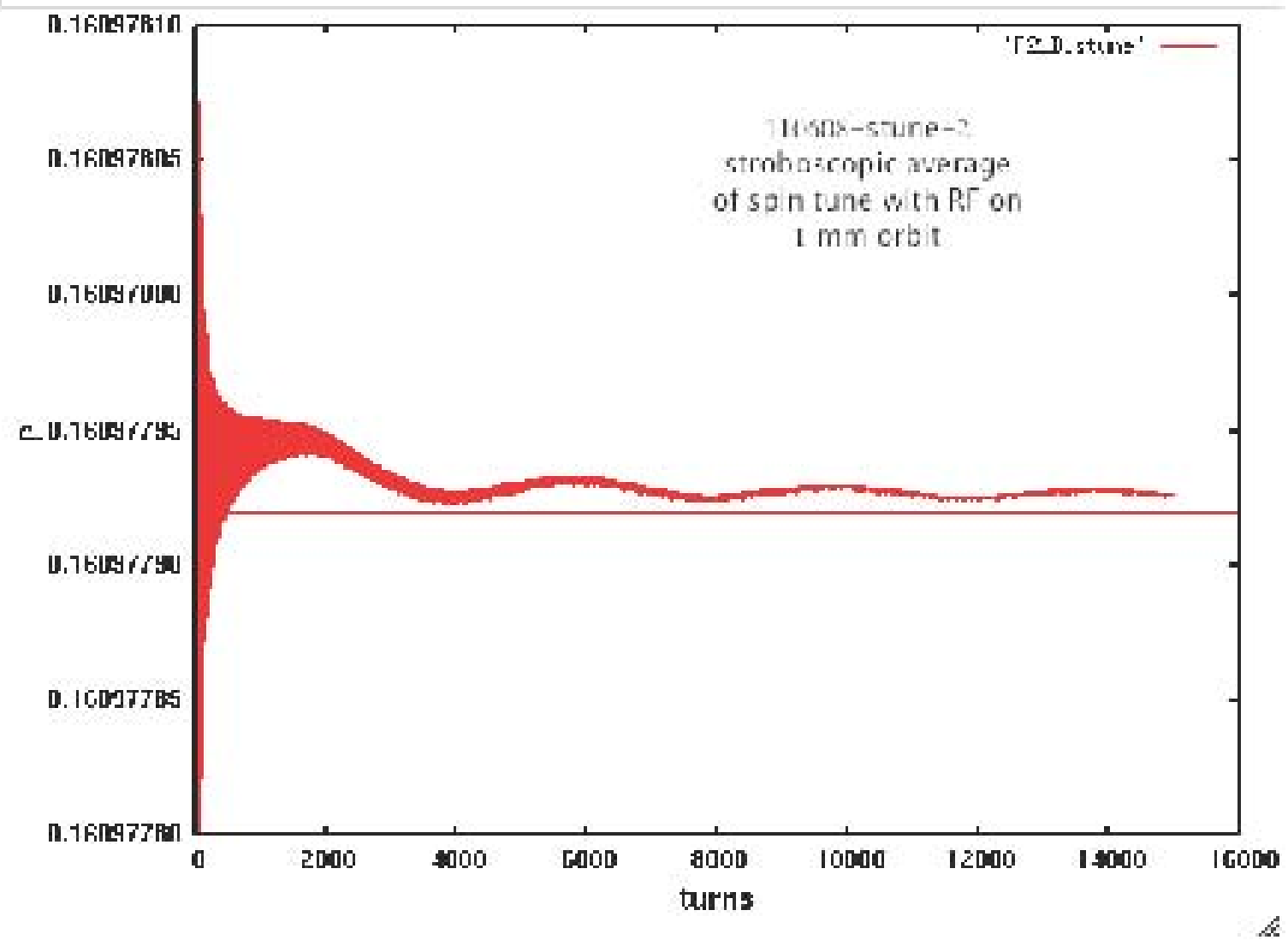

Figure 6: Spin tune strobo average in the presence of synchrotron oscillation. The synchrotron period is about 4,000 turns. Plot for a particle at $d p / p=$ $3.10^{-4}$ near the edge of the bucket. RF parameters were $V=400 \mathrm{~V}, h=1$. The horizontal line is $G \gamma$. 


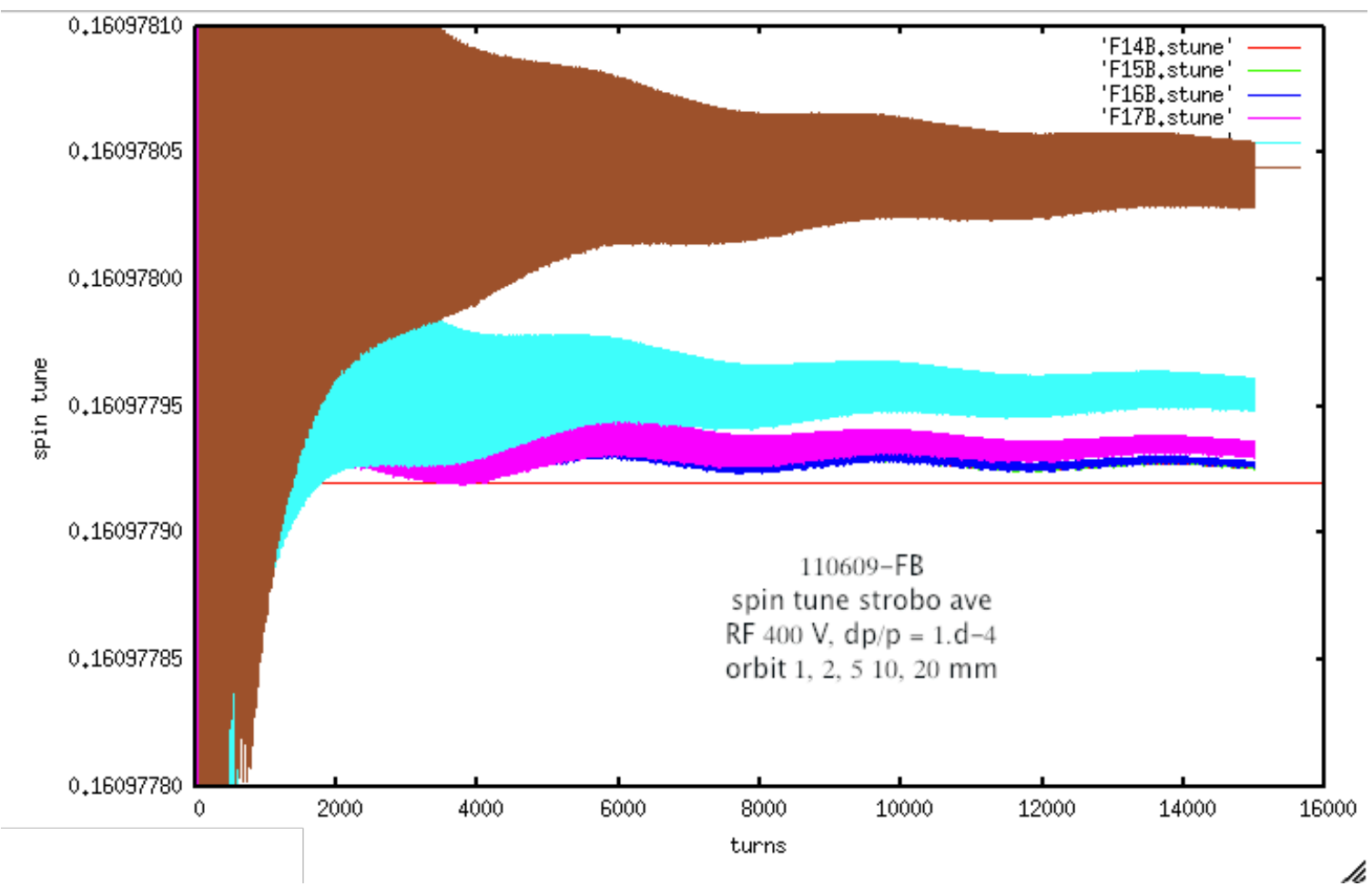

Figure 7: Spin tune with RF motion combined with transverse motion. Orbit for $1,2,5,10,20 \mathrm{~mm}$ 
Results for spin tune are in the following table with Synchro- and Betatron motion combined. The spin tune has been calculated as the average of the oscillatory pattern.

\begin{tabular}{lll} 
to Fig.7 & & $Q_{\text {spin }}$ \\
\hline $0 \mathrm{~mm}$ & $:$ & 0.16097792969857347 \\
$1 \mathrm{~mm}$ & $:$ & 0.16097793022338133 \\
$2 \mathrm{~mm}$ & $:$ & 0.16097793134006960 \\
$5 \mathrm{~mm}$ & $:$ & 0.16097793805215579 \\
$1 \mathrm{~cm}$ & $:$ & 0.16097796094671354 \\
$2 \mathrm{~cm}$ & $:$ & 0.16097805034758250 \\
$5 \mathrm{~cm}$ & $:$ & 0.16097867426109169 \\
\hline
\end{tabular}

The largest difference in spin tune in this table is $1.710^{-6}$, with an average spin tune line width of the order of $1.10^{-6}$, corresponding to an uncorrected spin coherence of the order of $10^{6}$ turns, only limited by betatron and synchrotron motion.

In conclusion, indications are that with an energy spread not exceeding the bucket allowance of $3.10^{-4}$ the spin tune line width for the present configuration of COSY, even before sextupoles were activated, and before cooling, is of the order of $10^{-7}$.

Figs.9 and 10 show the spin tune vs. orbit amplitude for the cases just described. The curves for the tune are quadratic in the transverse coordinates, therefore they can be corrected by sextupoles, in order to obtain the same spin tune for all particles sampled, and improve the spin coherence, as it was performed in the experiments.

In the simulation the 18 sextupoles available in COSY (see Fig.8 were all set at the same strength. Fig.9 shows the spin tune for deuterons injected at different transverse amplitudes $x$ and $y$. In this case the correction was achieved with sextupoles set at $K_{2} L=0,00063 \mathrm{~T} / \mathrm{m}$. Fig. 10 shows the spin tune with particle injected at different vertical $y$ amplitudes and $x=0$. In this case spin coherence correction was achieved with the smaller sextupole strength of $K_{2} L=0,00037 \mathrm{~T} / \mathrm{m}$, because only a partial correction is needed. For these cases the RF cavity and synchrotron motion were on and . In both cases for all particles in the simulation the energy spread was $d p / p=3.10^{-4}$ near the edge of stability in the bucket. Also the RF solenoid was on, kept at the resonance frequency. 


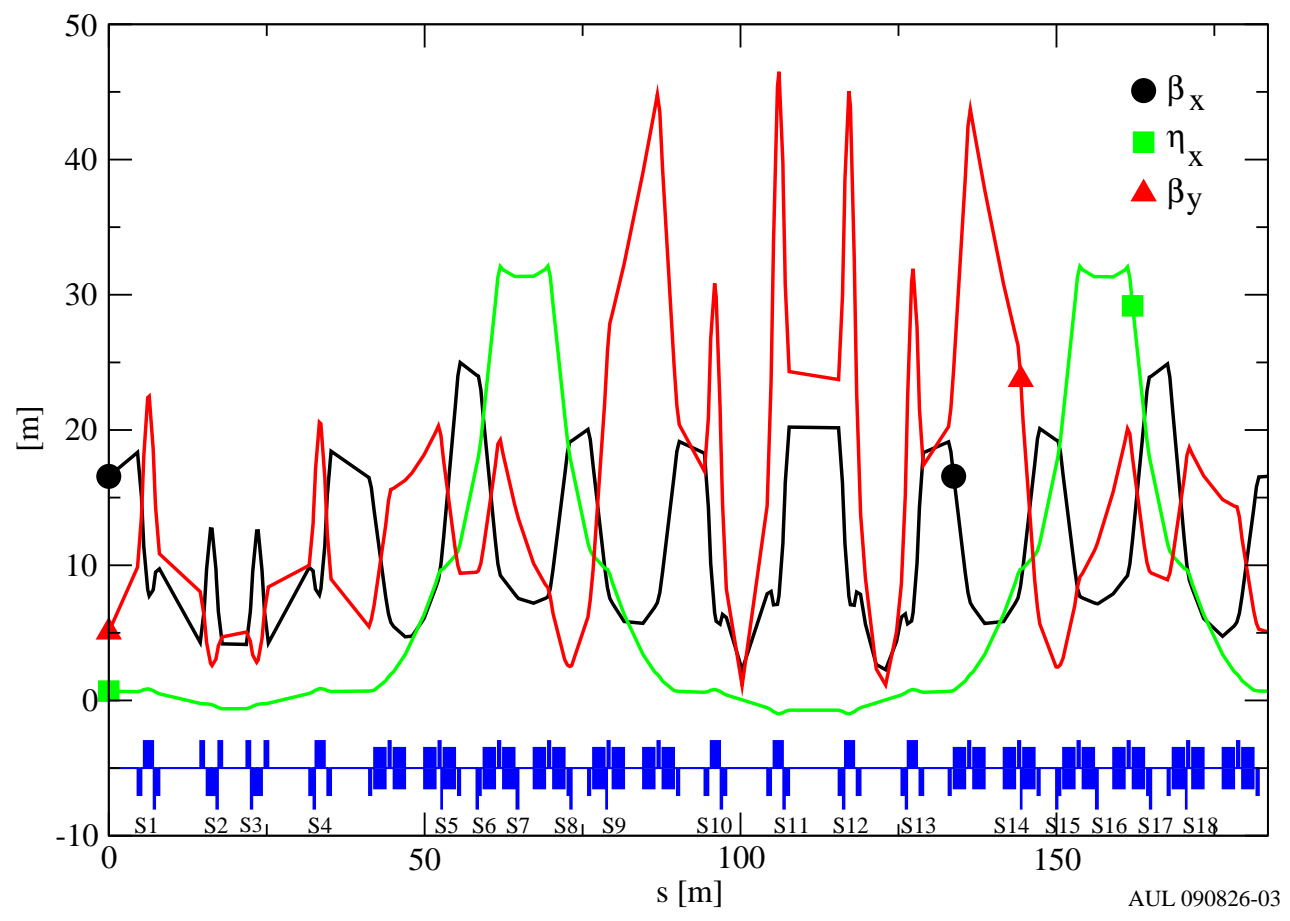

Figure 8: Lattice of COSY and Twiss functions. The position of the 18 sextupoles is indicated 


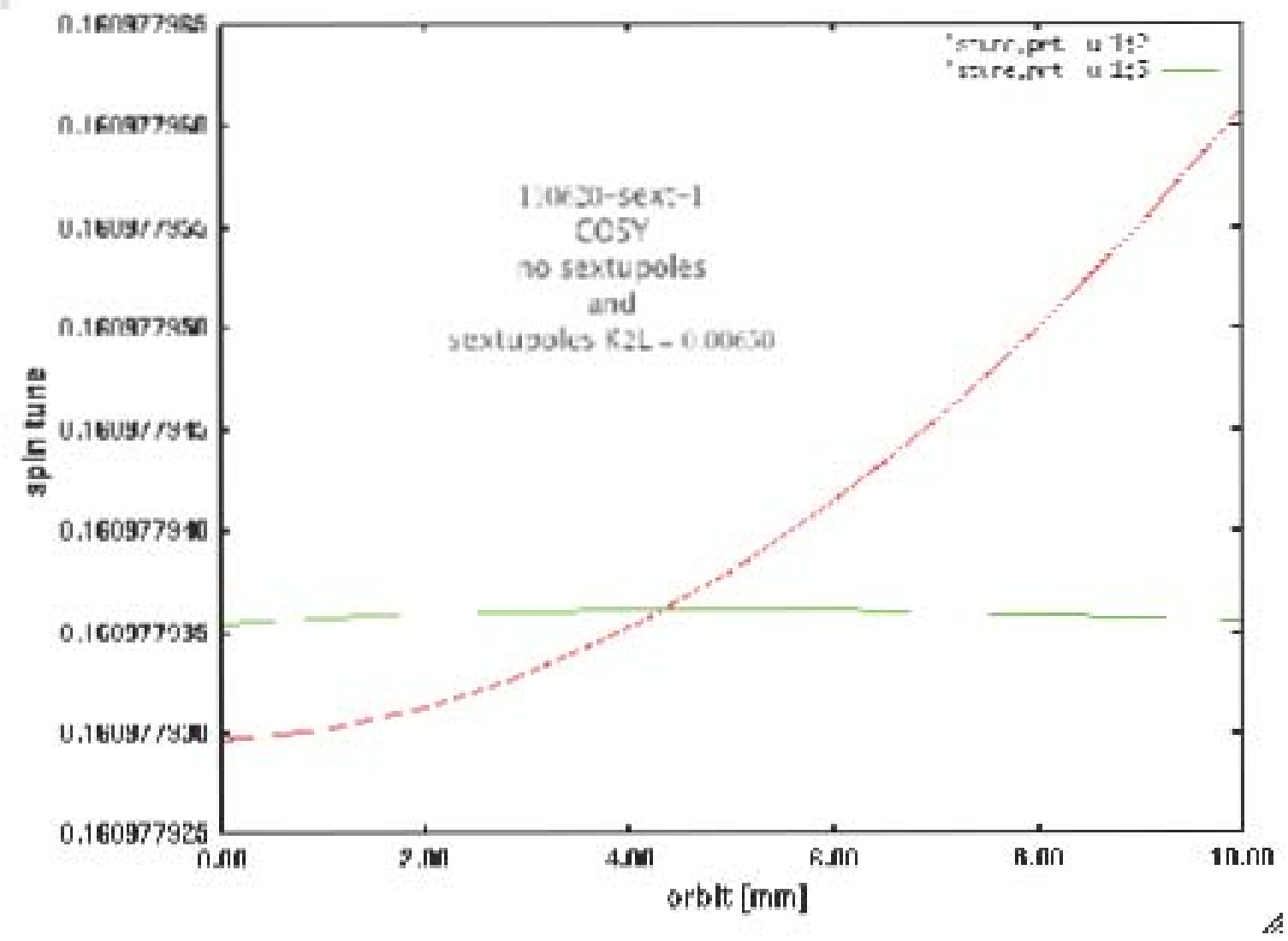

Figure 9: Spin tune vs. orbit amplitude in both $x$ and $y$ for the cases of FIGS.6 and 7. Correction of spin coherence by means of sextupoles is shown. Uncorrected Spin tune in the $4.10^{-8}$ range. 


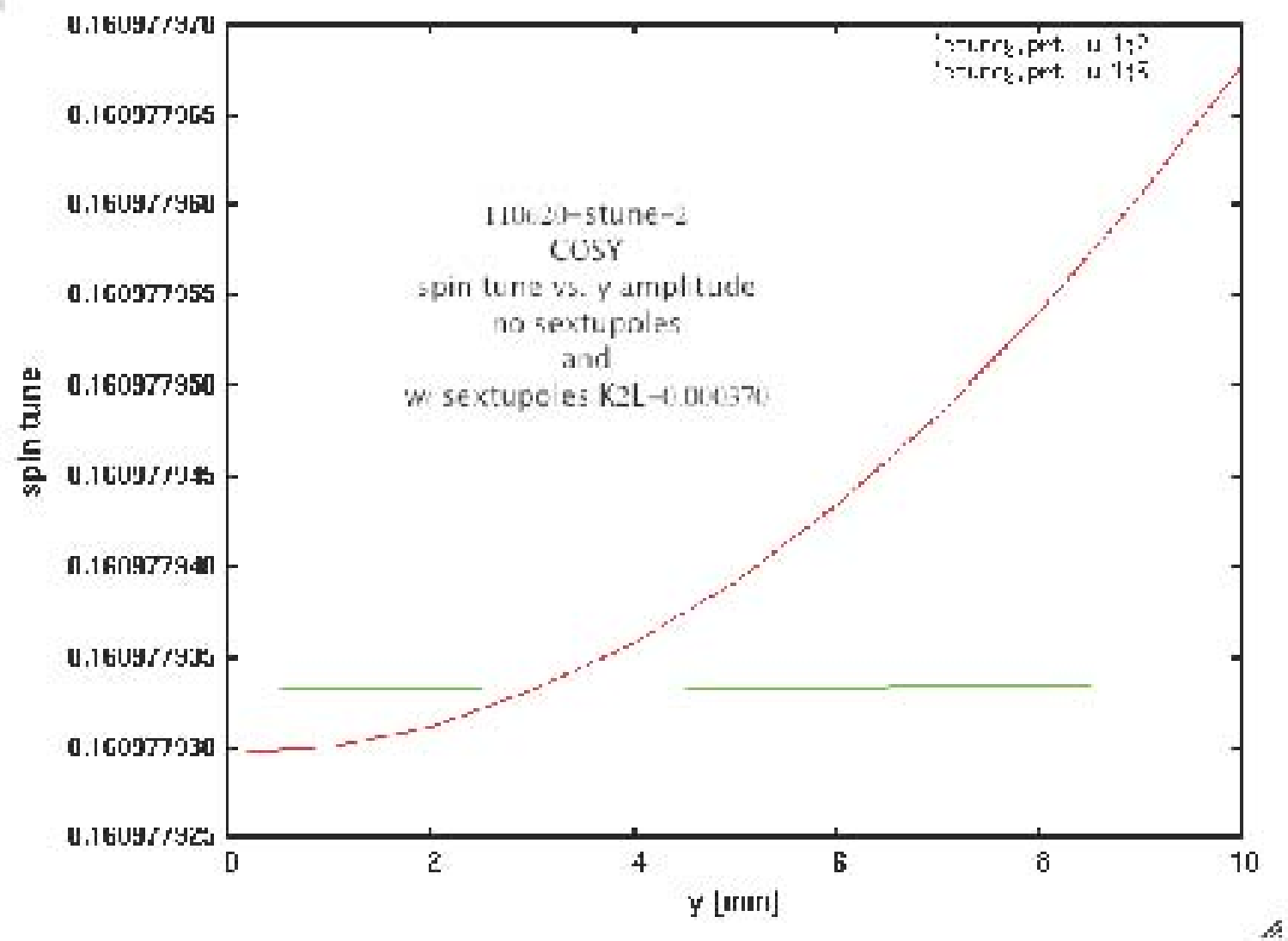

Figure 10: Spin tune vs. orbit amplitude only in the vertical $y$. Correction by sextupoles is also shown. Uncorrected Spin tune in the $5.10^{-8}$ range. 


\section{References}

[1] Y.K.Semertzidis (Spokeperson) Search for a permanent electric dipole moment of the deuteron nucleus at the $10^{-28}$ e.cm level AGS Proposal, BNL (2008) yannis@bnl.gov

[2] F. Méot Zgoubi URL: SourceForge.net/projects/zgoubi URL: unwphysics.umd.edu/dsat/dsatmarylie.html

[3] N.Malitsky and R.Talman UAL: Unified Accelerator Library BNL71010-2003, formal report

URL: http://nuw.bnl.gov/lrd/documents/24937.pdf

[4] A.U.Luccio Spink User's Manual Brookhaven National Laboratory

[5] RHIC Relativistic High Ion Collider Brookhaven National Laboratory URL: http://www.bnl.gov/rhic

[6] R.Talman and L.Schlachinger Teapot: A Thin Element Accelerator program for Optics and Tracking Part.Accel. 22,35 (1991)

[7] A.U.Luccio, F.Lin, G.Onderrwater, E.J.Stephenson Tracking studies of spin coherence in COSY in view of EDM polarization measurements Proc. PST2009, Ferrara, Italy, 6-11 Sept 2009 